\title{
PERSPECTIVAS DE CONSUMO Y EMPLEABILIDAD \\ DE LA GENERACIÓN Z BASADOS EN SUS \\ GUSTOS MUSICALES
}

\author{
Raúl Ignacio Cruz Maldonado ${ }^{17}$ \\ Ingrid Patricia Ortega Patiño ${ }^{18}$ \\ Corporación Universitaria Minuto de Dios
}

\section{Resumen:}

Esta investigación tiene como propósito examinar la relevancia de la inclinación musical en la vida personal y su incidencia en las perspectivas de consumo y empleabilidad en la generación denominada Z (Di Lucca, 2013) de la localidad Rafael Uribe Uribe. En este sentido, identifica la presencia de la música en los hábitos, identidad cultural, expresión y aceptación de la comunidad. La inclinación musical podría ser un referente determinante para pronosticar el tipo de labores a desempeñar. Como metodología se realiza una recolección de datos para conocer y exponer los tipos de géneros musicales más influyentes en el comportamiento de esta generación, metas personales, estilo de vida y las preferencias que pueden cambiar por el tipo de género que escuchan. La presente investigación es de carácter exploratoria con base cualitativa; se indagan los diferentes géneros musicales y las horas dedicadas a esta actividad, en contraste con la proyección de labor a desempeñar. En el apartado de conclusiones se presentan las tendencias de preferencia reflejadas en una matriz de impacto cruzado: preferencia vs género musical.

\section{Palabras clave:}

perspectiva; empleabilidad; consumo; generación Z; gustos musicales.

\footnotetext{
${ }^{17}$ Investigador docente. Correo electrónico: raul.cruzmaldonado@uniminuto.edu.co

${ }^{18}$ Estudiante del semillero de investigación Tecnología de Gestión de Mercadeo. Correo electrónico: iortegapati@uniminuto.edu.co
} 


\section{Abstract:}

This research aims to examine the relevance of the musical inclination in personal life and the impact of this on the perspectives of consumption and employability in the generation called Z (Di Lucca, 2013), from the Rafael Uribe Uribe locality, as it Identify that music is present in the habits, cultural identity, expression and acceptance of the community. The musical inclination could be a determining reference to predict the type of work to be performed. As a methodology, we will carry out a data collection, which will allow us to know and mention which type of musical genres are more influential in the behavior of this generation; personal goals, lifestyle, and preferences that may change for the type of gender they hear. The investigation is with a qualitative approach, type exploratory, its analyze the different musical genres and the hours dedicated to this activity are investigated in contrast to the projection of work to be performed. The conclusions section will show what preference trends are reflected in a cross-impact matrix; Preference vs. musical genre, the relevance is given since the work performance approach determines the finances of an individual and territory.

\section{Keywords:}

perspective, employability, consumption, Generation Z, Musical Tastes.

\section{Introducción}

A continuación, se presenta la introducción de la investigación acerca de la influencia que tienen los gustos musicales en la vida, las perspectivas de consumo y la empleabilidad de la generación Z en la localidad Rafael Uribe Uribe. Dicha generación se ve motivada a crear un estilo de vida relacionado al tipo de música que escuchan, como por ejemplo el reggaetón. Incluso aunque los jóvenes tengan una formación ética y moral desde sus hogares, la exposición y auge de este género musical tiende a provocar e invadir sentimientos relaciona- 
dos con el tipo de letra contenido en cada canción. Como bien es conocido este género, dentro de sus letras, incluye expresiones denigrantes hacia la mujer; además hacen referencia al dinero fácil, fama, vehículos y casas de alta gama, drogas e incluso sexo.

Este género musical habla sobre contenidos sexuales, sobre droga y denigra a la mujer hasta tal punto que la ven como una esclava de sus movimientos y como un objeto sexual.

Los jóvenes, aunque suelen pasar por alto los temas sexuales de estas letras, no quiere decir que no se vean influenciados por las mismas. Los adolescentes son los más propensos a estas influencias, no solo del reggaetón sino también de los medios de comunicación en general, porque su personalidad se basa en elementos como la forma de vestir, de hablar, sus gustos musicales y la posesión de objetos materiales que coinciden con los de sus amigos. De esta manera forman grupos que tienen los mismos gustos y, a partir de esto, va a depender su conducta, valores e identidad (Caladín, 2017).

La necesidad de indagar y reflexionar sobre este tema surge de la evidente preocupación de los padres y sociedad; existen niños, adolescentes y jóvenes cambiando su forma de ver el mundo por lo que escuchan. Dentro de los diferentes sitios web consultados, se consigue un dato relevante en relación con la edad (desde los 6 años hasta los 12) y la influencia del género. A continuación, el resultado.

La etapa que va de los 6 a los 12 años es muy importante en cuanto la asimilación de valores y patrones sociales. Los niños comienzan a entender el significado de ciertos valores: solidaridad, empatía, amistad. Para asimilarlos, buscan un patrón de referencia. Los padres suelen ser los primeros en los que los niños se contemplan, pero, ¿qué ocurre cuando de pronto se sienten absorbidos por mensajes muy diferentes que le llegan a través de canciones como el reggaetón o el trap? Que los absorben igualmente. El daño que les puede causar es mayor del que imaginamos (Muñoz, 2017). 
La búsqueda de información evidencia que la mejor edad para que no afectar el desarrollo cognitivo de los niños es después de los 12 años. En ese momento van definiendo su personalidad y es posible que los valores éticos y morales, infundidos por los padres, no se vean mal influenciados por las letras de las canciones que cada vez son más cargadas de contenido obsceno, que incluso una persona de mayor edad considera demasiado vulgar. Cabe resaltar que la influencia de los artistas no es solo por medio de sus letras, también por medio de redes sociales como Instagram, Facebook, Snapchat. Allí los menores de edad tienen acceso y pueden observar con mayor cercanía la forma de vestir, sus actividades cotidianas, la exposición de su vida personal e incluso los escándalos en los que se puedan llegar a ver expuestos.

Esta investigación se realiza con el ánimo de crear un referente en el mundo académico que aborde los temas del mercado laboral desde características etnográficas. Además, es un aporte para aquellos estudiantes de áreas dedicadas a las ciencias sociales, para dar continuidad al tema o investigar temáticas similares. Es de carácter exploratorio, con base cualitativa; se indagan cuestiones como el tipo de música y la cantidad de horas dedicadas a esta actividad en contraste con la proyección de labor a desempeñar. Los resultados son trabajados a partir de pruebas estadísticas del rango exploratorio y de acuerdo con el tamaño de la muestra, algunos de carácter inferencial.

Después del análisis de los datos se espera recolectar información acerca del género musical que prefiere, tres (3) profesiones que gustaría estudiar profesionalmente, cuánto espera de ingresos, en qué edad espera obtener esos ingresos, entre otros.

\section{Consideración final}

La siguiente fase de la investigación presentará las tendencias de preferencia a través de una matriz de impacto; cruzará la preferencia vs género musical. El alcance de la pertinencia reside en que a partir del enfoque de desempeño laboral se determinan las finanzas de un individuo y de su territorio. 


\section{Referencias bibliográficas}

Borbolla, K. (2017). ¿Qué es el trap y por qué se considera música "sucia"? Debate. Recuperado de: https://www.debate.com.mx/Quees-el-trap-y-por-que-se-considera-musica-sucia-t201710220001.html

Cabrera, I. M. (2013). Influencia de la música en las emociones: una breve revisión. Realitas: revista de Ciencias Sociales, Humanas y Artes, 1(2), 34-38.

Caladin, A. (2017). Influencia del reggaetón en los jóvenes. Amparo Paladín Psicólogos. Recuperado de: http://www.amparocalandinpsicologos.es/influencia-del-reggaeton-en-los-jovenes/

Di Lucca, S. (2013). El comportamiento actual de la Generación Z en tanto futura generación que ingresará al mundo académico. Categoría: Proyectos de Exploración de la Agenda Profesional, 1-33.
Gallucci, M. J. (2008). Análisis de la imagen de la mujer en el discurso del reggaetón. Opción, 24(55), 84-100.

Muñoz. (2017). El reggaetón es perjudicial para el desarrollo cognitivo de los niños. Guía Infantil. Recuperado de: https://www.guiainfantil.com/blog/educacion/valores/el-reggaeton-es-perjudicial-para-el-desarrollo-cognitivo-de-los-ninos/

Massot, D. (2018). Generación Z: ¿conocen las 15 características de los nacidos entre 1995 y 2010? Aleteia. Recuperado de: https:// es.aleteia.org/2018/04/09/generacion-z-conocen-las-15-caracteristicas-de-los-nacidos-entre-1995y-2010/ 